\title{
Systematic review and meta-analysis of diagnostic test accuracy (DTA) studies: the role of cerebral perfusion imaging in prognosis evaluation of mild cognitive impairment
}

\author{
Ling Zhu ${ }^{1}$, Wenjuan $\mathrm{Zhao}^{2}$, Jun Chen ${ }^{3}$, Guifen $\mathrm{Li}^{2}$, Jibo $\mathbf{Q u}^{2}$ \\ ${ }^{1}$ Department of Neurology, Yunnan Honghe Prefecture Central Hospital (The Fifth Affiliated Hospital of Kunming Medical University), Gejiu, \\ China; ${ }^{2}$ Department of Neurology, Southern Central Hospital of Yunnan Province (Hospital of Honghe State Affiliated to Kunming Medical \\ University), Mengzi, China; ${ }^{3}$ Department of Neurology, Non Affiliated The People's Hospital of Mengzi, Yunnan University of Chinese Medicine, \\ Mengzi, China \\ Contributions: (I) Conception and design: L Zhu, J Qu; (II) Administrative support: W Zhao; (III) Provision of study materials or patients: L Zhu, \\ W Zhao, J Chen, G Li; (IV) Collection and assembly of data: All authors; (V) Data analysis and interpretation: L Zhu, J Chen, G Li, J Qu;(VI) \\ Manuscript writing: All authors; (VII) Final approval of manuscript: All authors. \\ Correspondence to: Jibo Qu. 100 meters south of Shanghai Road at the intersection of Honghe Avenue and Shanghai Road, Honghe Prefecture, \\ Mengzi, China. Email: yunnanhhqjb@126.com.
}

Background: The application value of cerebral perfusion imaging, such as fluorodeoxyglucose-positronemission computed tomography (FDG-PET), single-photon emission computed tomography (SPECT), and structural magnetic resonance imaging (MRI), in the prognostic assessment of mild cognitive impairment (MCI) remains unclear. Thus, it was the focus of this meta-analysis, which aimed to provide a theoretical basis for early diagnosis of neurological diseases.

Methods: The Boolean logic retrieval method was used to retrieve related literature, with "cognitive disorder", "cerebral perfusion imaging”, "cerebral imaging”, "mild cognitive impairment”, and "prognostic assessment” as search terms. The PubMed, Medline, CNKI (China National Knowledge Infrastructure), and other databases were searched from the date of establishment of the database to December 30, 2020 for literature on the prognostic assessment of MCI using FDG-PET, SPECT, and MRI imaging. Meta-analysis was performed using Review Manager software.

Results: A total of 16 references were identified, most of which had a low risk of bias (i.e. medium and high-quality). The meta-analysis results showed that the sensitivity and specificity of FDG-PET imaging was significantly higher than those of SPECT and MRI imaging, and the differences were statistically significant $(\mathrm{P}<0.05)$. The summary receiver operating characteristic (sROC) curves for the sensitivity and specificity of FDG-PET, SPECT, and MRI imaging did not present a "shoulder arm" distribution, and there was no threshold effect.

Discussion: Cerebral perfusion imaging has good prognostic value for patients with MCI, and FDG-PET imaging has better predictive ability of the prognosis for patients with MCI.

Keywords: Cerebral perfusion imaging; cognitive impairment; computed tomography; prognosis evaluation

Submitted Dec 11, 2021. Accepted for publication Feb 16, 2022.

doi: 10.21037/apm-21-3916

View this article at: https://dx.doi.org/10.21037/apm-21-3916 


\section{Introduction}

Mild cognitive impairment (MCI) refers to a mild decline in cognitive functions such as memory, orientation, thinking judgment, spatial configuration, and language. Although MCI has not yet affected the ability to perform daily living, it may develop into dementia without intervention (1). Studies have shown that MCI is a precursor to dementia, with more than $10 \%$ of people with MCI converting to dementia each year. Only 1 to 2 percent of normal older adults develop dementia each year, so MCI is at high risk of developing dementia. Once detected and diagnosed, it is important to actively treat the disease. There are many causes of MCI, including neurodegenerative diseases such as Alzheimer's disease, Parkinson's disease, frontotemporal degeneration, and cortical basal ganglia degeneration (2). Vascular factors (such as cerebral infarction, cerebral thrombosis, and cerebral hemorrhage), infections (such as autoimmune encephalitis, viral encephalitis, and limbic encephalitis), and nutrient/vitamin deficiency and metabolic dysfunction (such as hypothyroidism) (3). If the above factors can be effectively controlled, cognitive dysfunction may not progress and can be stabilized or even restored to normal. The main clinical manifestations of MCI is memory impairment, and it is the short-term memory that declines first; that is, daily activities and some commonly used items are often forgotten (4). Cerebral blood perfusion disorder is one of the high risk factors for the onset and progression of cognitive dysfunction. Cerebral blood perfusion imaging is helpful to discover hemodynamic changes in patients with cognitive dysfunction and dementia, so as to diagnose the disease.

Study found that timely intervention in patients with mild cognitive dysfunction can delay the progress of dementia, help patients keep positive and optimistic attitude, so as to effectively improve memory function in patients with MCI, currently on the intervention of the disease including nonspecific intervention, cognitive training, drug intervention and regulate lifestyle (sleep), control diet led, etc. (5). In order to reveal the cause of MCI or associated with disease, besides neuropsychological assessment of patients, also suggested that detection of hematology, including blood counts, blood sedimentation, blood electrolyte, thyroid hormone levels, etc., in addition, some patients still need to improve the head CT (computer tomography) scan, head magnetic resonance (MRI) scan imaging examination, etc. Brain perfusion imaging (BRAIN perfusion imaging) refers to the diagnosis of brain diseases using radioactive drugs that can pass the normal blood-brain barrier into the body and obtain clear images reflecting brain function and metabolism through imaging instruments. Cerebral perfusion imaging is mainly used to evaluate the state of cerebral blood flow and metabolism. Together with cerebral vascular anatomical structure examination represented by total cerebral angiography, it sketches the original appearance of cerebrovascular disease. It is mainly used to evaluate cerebral ischemic focus and collateral circulation clinically, and the gold standard for judging ischemia is cerebral oxygen metabolism rate (6). Cerebral perfusion imaging agent into the amount of brain cells is proportional to the local cerebral blood flow, using brain perfusion imaging, after image reconstruction can obtain the transverse, coronal and sagittal plane image, according to the brain, the cerebellum, basal ganglia and brain stem parts local blood flow imaging, according to a certain number of physical model can calculate the various parts of the local blood flow and average whole brain blood flow.

Relevant studies have shown that cerebral perfusion imaging can be used to evaluate the local hemodynamic changes of hematoma and its surrounding areas, thus assisting in the diagnosis and prognosis of cerebral hemorrhage. To some extent, for the diagnosis of brain diseases, besides total cerebral angiography, many patients need cerebral perfusion imaging, especially for ischemic cerebrovascular disease, so as to have a comprehensive and in-depth understanding of the disease. Therefore, this study adopted the meta-analysis method to study the application value of cerebral perfusion imaging in the treatment prognosis evaluation of patients with MCI.

We present the following article in accordance with the PRISMA-DTA reporting checklist (available at https://apm. amegroups.com/article/view/10.21037/apm-21-3916/rc).

\section{Methods}

\section{Literature retrieve}

The Boolean logic retrieval method was adopted to select relevant literatures, with "cognitive disorder", "cerebral perfusion imaging", "cerebral imaging", "mild cognitive impairment", "prognostic assessment" as search terms. PubMed, Medline, EMbase, China Biomedical Literature Database, CNKI database, Wanfang Database, VIP database, and Google Scholar were searched for literature on the prognostic evaluation of MCI using cerebral perfusion imaging. The retrieval time was from the establishment of the database to December 30, 2020. The 
search terms were combined freely, and after several rounds of searching to determine the literature, the search engine was used to trace the identified references. The quality of the literature was evaluated using RevMan 5.2 software provided by Cochrane system.

\section{Literature inclusion and exclusion criteria}

The literature to be included was required to meet all of the following criteria: (I) the study subjects were patients diagnosed with MCI without pathological type limitation; (II) prognostic assessment was conducted through cerebral perfusion imaging, including fluorodeoxyglucose-positronemission computed tomography (FDG-PET), singlephoton emission computed tomography (SPECT), and structural magnetic resonance imaging (MRI); and (III) study types: diagnostic accuracy test.

References that met any of the following criteria were excluded: (I) the included subjects had severe infections; (II) repeated literature and studies with too few experimental samples; (III) invalid or missing data; and (IV) overlapping research subjects or data.

Two senior experts independently carried out the screening of the abstracts and the full texts, and three preexperiments were conducted before screening. Disagreements were resolved through discussion or arbitration by a third expert.

\section{Quality assessment}

In this study, the Newcastle-Ottawa Scale (NOS) of the Cochrane Collaboration was used to evaluate the literature quality, and a star system was used for measurement, factoring in the subjects, case comparison, and inter-group comparison (the total score was 9). Studies with a score of $\geq 7$ stars were considered high quality; that is, had a low risk of bias. Studies with $\leq 1$ star were considered low quality; that is, had a high risk of bias. Studies with 2-6 stars were considered medium quality; that is, had a medium risk of bias.

Quality evaluation of the references was carried out independently by two experts, and three pre-experiments were carried out before the evaluation. Disagreements were resolved through discussion or arbitration by a third expert.

\section{Data extraction}

Two experts used a unified Excel table for independent extraction of data, and three pre-experiments were carried out before extraction. Disagreements were resolved through discussion or arbitration by a third expert. The following data were collated: (I) first author and publication year; (II) age of enrolled patients; (III) follow-up time and mental state scores of patients; (IV) the progression of the patient's cognitive dysfunction (whether it progressed to Alzheimer's disease).

\section{Statistical analysis}

Meta-analysis was performed using Review manager 5.3 software (provided by Cochrane International Collaboration Network for systematic review workers). The mean deviation (MD) or standard mean deviation (SMD) and $95 \%$ confidence interval (CI) were used for efficacy analysis statistics. The heterogeneity test ( $Q$ test) was first performed on the included studies. The risk of bias of the included references was assessed using Review Manager software. Each effect was expressed using the $95 \% \mathrm{CI}$. When $\mathrm{P}>0.1$ and $\mathrm{I}^{2}<50 \%$, the fixed-effect model (FEM) was used for meta-analysis; however, when $\mathrm{P}<0.1$ and $\mathrm{I}^{2}>50 \%$, the random-effects model (REM) was used.

\section{Results}

\section{Literature retrieve and NOS results}

As shown in Figure 1, a total of 527 references were initially retrieved. Of these, 136 were excluded after reading the titles and abstracts. A further 148 references were removed by reading the full texts, and finally 16 references [including five FDG-PET (7-11), four SPECT (12-15), seven MRI (16-22) were included for meta-analysis]. Literature was excluded based on the following: subjects with primary acute and chronic cardiopulmonary dysfunction and serious mental diseases (40 articles); animal experiments (33 articles); overlapping subjects (37 articles); related information could not be extracted (45 articles); cerebral perfusion imaging was not performed on patients (63 articles); and lack of original data (66 articles). Tables 1-3 show the basic information of the included literature published from 2005 to 2020. Figure 2 displays the NOS results. Notably, there were seven references with $\geq 7$ stars or above, eight references with 2-6 stars, and one reference with $>2$ stars. All of the included articles were medium- and high-quality references. 


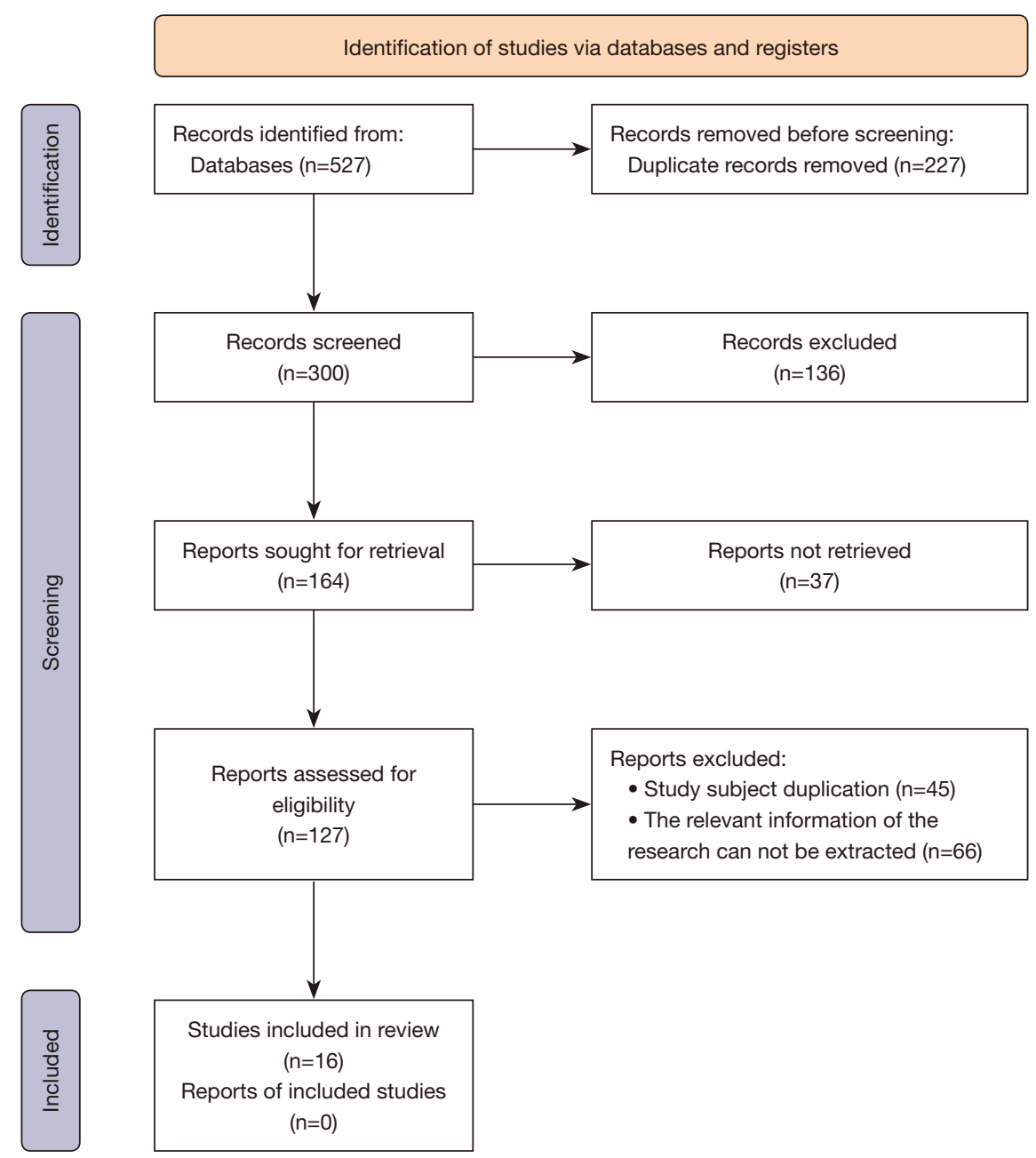

Figure 1 Literature screening flowchart.

Table 1 Basic information of the included literature (FDG-PET)

\begin{tabular}{|c|c|c|c|c|c|}
\hline Author & $\begin{array}{c}\text { Publication } \\
\text { year }\end{array}$ & Disease course & $\begin{array}{c}\text { Age } \\
\text { (years) }\end{array}$ & $\begin{array}{l}\text { Follow-up time } \\
\text { (months) }\end{array}$ & $\begin{array}{l}\text { Mental state } \\
\text { score }\end{array}$ \\
\hline Mosconi (7) & 2004 & Mild cognitive impairment to Alzheimer's disease & $67.1 \pm 7.7$ & 12 & $28.0 \pm 1.5$ \\
\hline Drzezga (9) & 2005 & $\mathrm{MCl}$ to dementia of the Alzheimer type (DAT) & $70 \pm 8$ & 15 & $26.9 \pm 1.9$ \\
\hline Arnáiz (11) & 2001 & $\mathrm{MCl}$ to Alzheimer's disease & $62.7 \pm 8.5$ & 36.5 & $26.9 \pm 2.3$ \\
\hline
\end{tabular}

FDG-PET, fluorodeoxyglucose-positron-emission computed tomography; $\mathrm{MCl}$, mild cognitive impairment. 
Table 2 Basic information of the included literature (SPECT)

\begin{tabular}{|c|c|c|c|c|c|}
\hline Author & $\begin{array}{c}\text { Publication } \\
\text { year }\end{array}$ & Disease course & Age (years) & $\begin{array}{l}\text { Follow-up time } \\
\text { (months) }\end{array}$ & $\begin{array}{c}\text { Mental state } \\
\text { score }\end{array}$ \\
\hline Encinas (12) & 2003 & $\mathrm{MCl}$ to Alzheimer's disease & $76.5 \pm 4.2$ & $\geq 12$ & - \\
\hline Hirao (14) & 2005 & Amnestic mild cognitive impairment to Alzheimer's disease & $69.0 \pm 8.6$ & 36 & $26.5 \pm 1.6$ \\
\hline Johnson (15) & 1998 & Suspected Alzheimer's disease $\rightarrow$ Alzheimer's disease & 72.5 & $\geq 24$ & - \\
\hline
\end{tabular}

SPECT, single-photon emission computed tomography; $\mathrm{MCl}$, mild cognitive impairment.

Table 3 Basic information of the included literature (MRI)

\begin{tabular}{|c|c|c|c|c|c|}
\hline Author & $\begin{array}{c}\text { Publication } \\
\text { year }\end{array}$ & Disease course & Age (years) & $\begin{array}{l}\text { Follow-up time } \\
\text { (months) }\end{array}$ & $\begin{array}{c}\text { Mental state } \\
\text { score }\end{array}$ \\
\hline Dickerson (16) & 2001 & $\mathrm{MCl}$ to Alzheimer's disease & $68.6 \pm 8.6$ & 12 & $27.0 \pm 2.2$ \\
\hline Convit (18) & 2000 & $\mathrm{MCl}$ to Alzheimer's disease & $74.0 \pm 7.0$ & 38 & $27.8 \pm 2.4$ \\
\hline Galton (21) & 2005 & Questionable dementia $\rightarrow$ Alzheimer's disease & $63.7 \pm 9.9$ & 24 & $27.4 \pm 2.4$ \\
\hline Teipel (22) & 2007 & Mild cognitive impairment to Alzheimer's disease & $69.7 \pm 8.5$ & 27 & $27.0 \pm 1.8$ \\
\hline
\end{tabular}

$\mathrm{MRI}$, magnetic resonance imaging; $\mathrm{MCl}$, mild cognitive impairment.

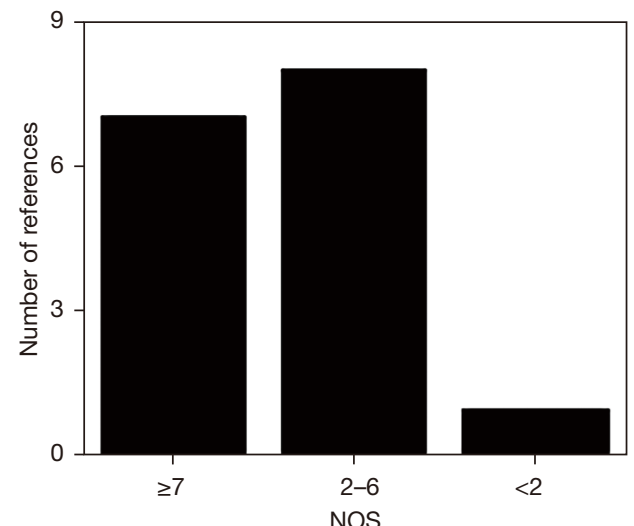

Figure 2 Newcastle-Ottawa Scale (NOS) results.

\section{Risk bias evaluation results}

Figures 3,4 show the multiple risk bias evaluations results of FDG-PET imaging, as drawn by Review Manager software. With each methodology feature considered, the evaluation results were input into the software to generate the bias-risk map. It was observed that the patient selection, test indicators, reference standards, and flow and timing in the FDG-PET references all had a low risk of bias, and that the low-risk bias for the blinding of subjects and researchers (implementation bias) was approximately $20 \%$, respectively. Except for the reference of Chételat et al. [2003] (8), the risk bias of the other literature was

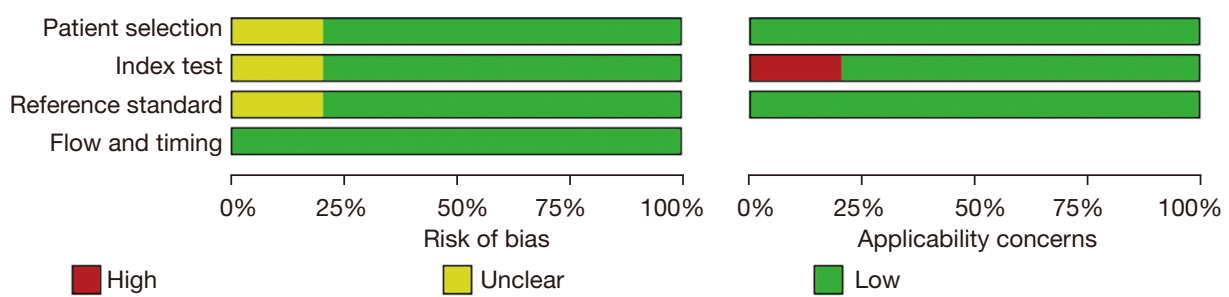

Figure 3 Bias risk evaluation results (FDG-PET). FDG-PET, fluorodeoxyglucose-positron-emission computed tomography. 


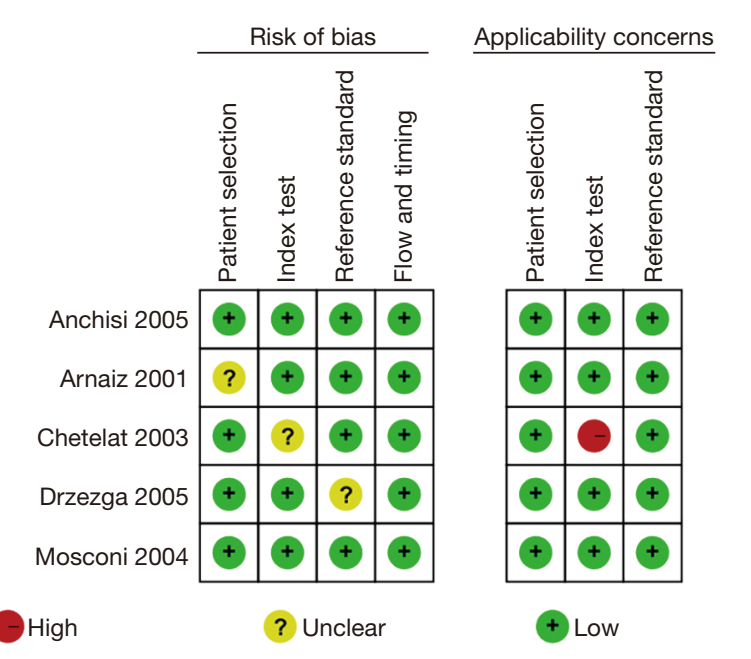

Figure 4 Multi-risk bias evaluation results (FDG-PET). FDG-PET, fluorodeoxyglucose-positron-emission computed tomography.

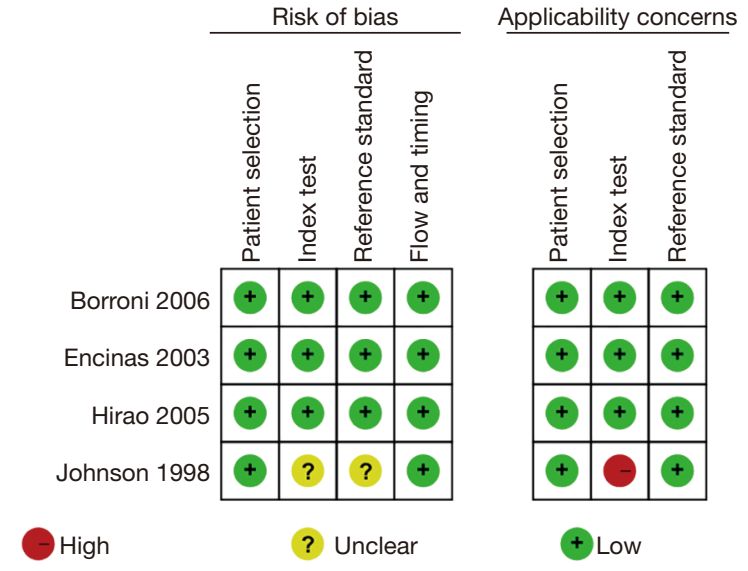

Figure 6 Multi-risk bias evaluation results (SPECT). SPECT, single-photon emission computed tomography.

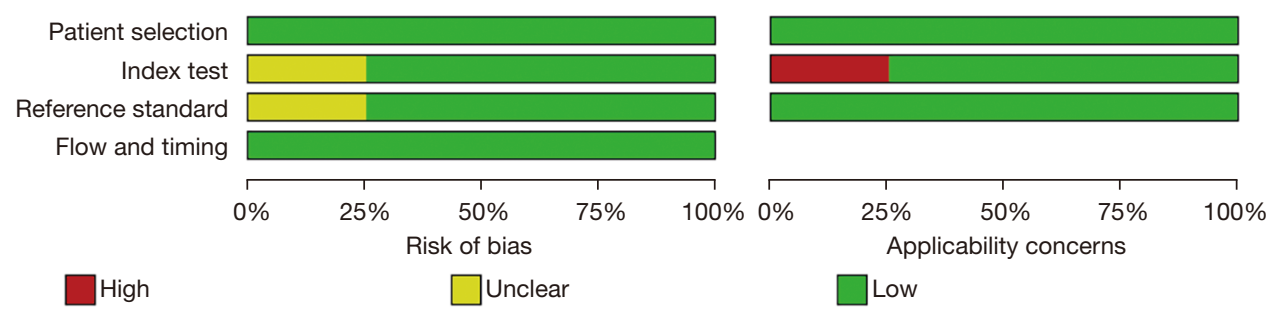

Figure 5 Bias risk evaluation results (SPECT). SPECT, single-photon emission computed tomography.

significantly low.

Figures 5,6 display the multiple risk bias evaluation results of patients using SPECT imaging, as drawn by Review Manager software. With each methodology feature considered, the evaluation results were input into the software to generate the bias-risk map. It was found that the patient selection, test indicators, reference standards, and flow and timing in the SPECT studies all had a low risk of bias, and that the low-risk bias for the blinding of subjects and researchers (implementation bias) was approximately $20 \%$, respectively. Except for the reference of Johnson et al. [1998] (15), the risk bias of the other literature was significantly low.

Figures 7,8 show the multiple risk bias evaluation results of MRI imaging, as drawn by Review Manager software. With each methodology feature considered, the evaluation results were input into the software to generate the bias-risk map. It was found that the patient selection, test indicators, reference standards, and flow and timing in the MRI studies all had a low risk of bias, and that the low-risk bias for the blinding of subjects and researchers (implementation bias) was approximately $15 \%$, respectively. Except for the reference of Convit et al. [2000] (18), the risk bias of other literature was significantly low.

\section{Sensitivity and specificity of the three imaging methods}

Tables 4-6 display the sensitivity and specificity of FDGPET, SPECT, and MRI imaging, respectively. It was found that the sensitivity, specificity, and positive likelihood ratio of FDG-PET imaging were significantly higher than those of SPECT and MRI imaging, and the differences were statistically significant $(\mathrm{P}<0.05)$.

\section{sROC curve of cerebral perfusion imaging}

Figures 9-11 were the summary receiver operating characteristic (sROC) curves of sensitivity and specificity 


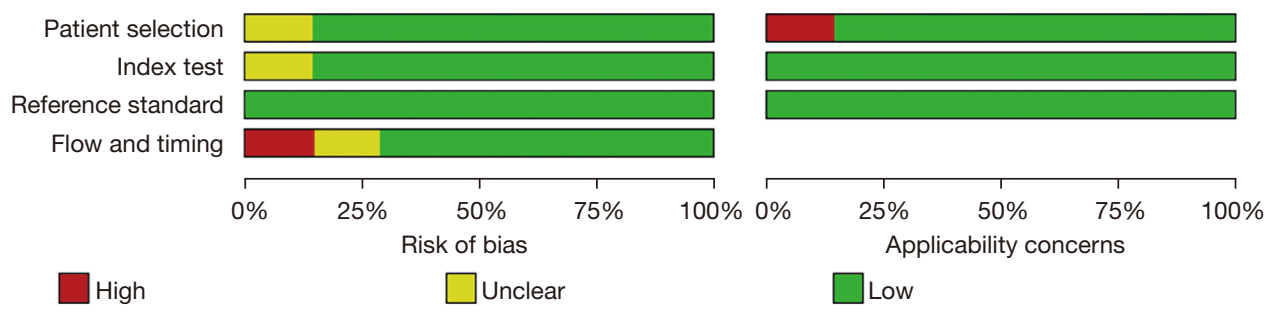

Figure 7 Bias risk evaluation results (MRI). MRI, magnetic resonance imaging.

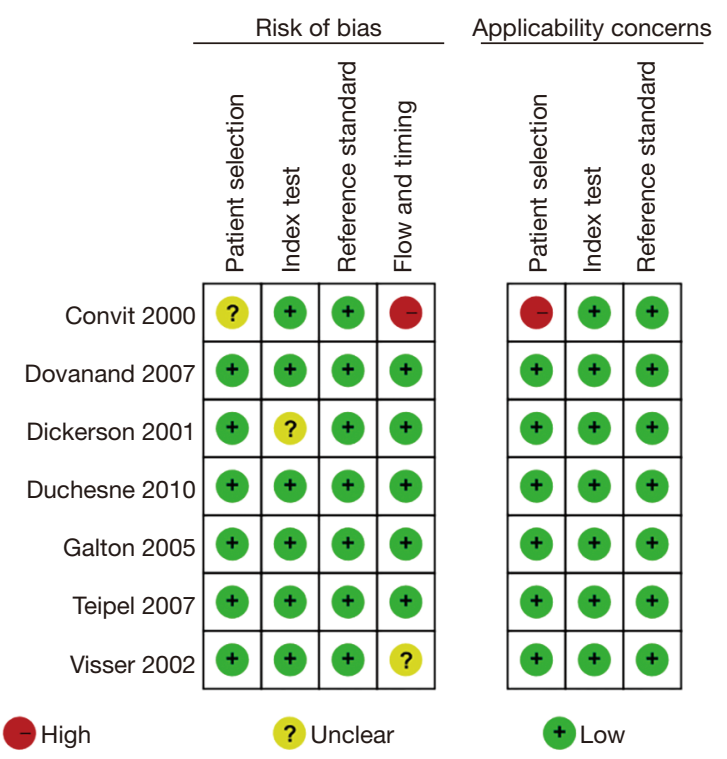

Figure 8 Multi-risk bias evaluation results (MRI). MRI, magnetic resonance imaging.

for FDG-PET, SPECT, and MRI imaging, respectively. It was observed that the sROC plane graph did not present a "shoulder-arm" distribution, and there was no threshold effect.

\section{Discussion}

Previous studies have shown that MCI is common in the elderly, and the risk of developing MCI increases with age (23). The cause of some MCI can be traced back, so it is necessary to go to the relevant outpatient clinic in time to understand the situation of cognitive impairment, judge whether it can be effectively treated, assess the risk of progression to dementia, and know the disease progress in detail $(24,25)$. More importantly, the basic data of patients' cognitive function should be kept to determine whether it has changed in the future. Early assessment is important because MCI is not a normal aging process and has a high risk of progression to dementia $(26,27)$. Regarding MCI assessment, it is important to understand the following: (I) subjective memory problems are not the basis of the diagnosis of MCI; (II) positive screening does not necessarily mean MCI; (III) MCI is not Alzheimer's disease; (IV) the diagnosis of MCI still requires further evaluation; and $(\mathrm{V})$ there are no reliable biomarkers for MCI (28).

In this meta-analysis, 16 articles were finally included. Of these, 14 adopted randomized controlled grouping, and only two used retrospective analysis, exhibiting bias. However, in general, this did not affect the results of this study. Meta-analysis was performed in this study to quantitatively combine each reference, which can not only avoid the sampling differences, but also expand the sample size and improve the credibility of the conclusion by assigning different weights to the references based on their results. The quality of meta-analysis mainly depends on the authenticity and integrity of the analyzed literature. Due to the objective factors, the number of included literature in this study was limited, so the sample size should be increased in subsequent studies to prevent bias in the analysis.

In this study, the application value of cerebral perfusion imaging in the prognosis assessment of MCI was investigated by meta-analysis of 16 references included in the Boolean logic retrieval method using FDG-PET, SPECT and MRI imaging respectively. The sensitivity and specificity positive likelihood ratio of FDG-PET imaging were significantly higher than those of SPECT and MRI imaging, the differences were statistically significant $(\mathrm{P}<0.05)$. This is similar to the results of Rane (29), indicating that cerebral perfusion imaging has better prognostic value for MCI, and FDG-PET imaging has better prognostic ability and effect for patients with MCI. In summary, SPECT, DG-PET, and MRI imaging have their own advantages and disadvantages in 
Table 4 Meta-analysis of FDG-PET imaging

\begin{tabular}{lcccc}
\hline Index & Value & $95 \% \mathrm{Cl}$ & $\mathrm{P}$ & $\mathrm{I}^{2}$ \\
\hline Sensitivity (\%) & 87.2 & $81.3-92.1$ & 0.013 & $65.9 \%$ \\
Specificity (\%) & 89.35 & $77.6-91.8$ & 0.036 & $56.8 \%$ \\
Positive likelihood ratio & 5.973 & $3.15-6.72$ & 0.498 & $<0.1 \%$ \\
Negative likelihood ratio & 0.132 & $0.05-0.49$ & $<0.001$ & $80.93 \%$ \\
Odds ratio & 38.996 & $17.66-87.1$ & 0.748 & $<0.1 \%$ \\
\hline
\end{tabular}

FDG-PET, fluorodeoxyglucose-positron-emission computed tomography.

Table 5 Meta-analysis of SPECT imaging

\begin{tabular}{lcccc}
\hline Index & Value & $95 \% \mathrm{Cl}$ & $\mathrm{P}$ & $\mathrm{I}^{2}$ \\
\hline Sensitivity (\%) & 80.5 & $78.3-90.12$ & 0.006 & $63.1 \%$ \\
Specificity (\%) & 74.3 & $61.3-78.5$ & $<0.001$ & $71.9 \%$ \\
Positive likelihood ratio & 2.637 & $1.38-4.81$ & $<0.001$ & $79.98 \%$ \\
Negative likelihood ratio & 0.334 & $0.18-0.50$ & 0.291 & $18.11 \%$ \\
Odds ratio & 11.27 & $4.52-20.01$ & 0.331 & $24.33 \%$ \\
\hline
\end{tabular}

SPECT, single-photon emission computed tomography.

Table 6 Meta-analysis of MRI

\begin{tabular}{lcccc}
\hline Index & Value & $95 \% \mathrm{Cl}$ & $\mathrm{P}$ & $\mathrm{I}^{2}$ \\
\hline Sensitivity (\%) & 71.9 & $64.8-80.12$ & 0.594 & $0.1 \%$ \\
Specificity (\%) & 79.44 & $77.6-91.8$ & 0.089 & $40.1 \%$ \\
Positive likelihood ratio & 3.298 & $2.59-6.72$ & 0.301 & 0.844 \\
Negative likelihood ratio & 0.365 & $0.30-0.48$ & 0.521 & $<0.1 \%$ \\
Odds ratio & 11.751 & $5.99-16.38$ & $<.1 \%$ & \\
\hline
\end{tabular}

MRI, magnetic resonance imaging.

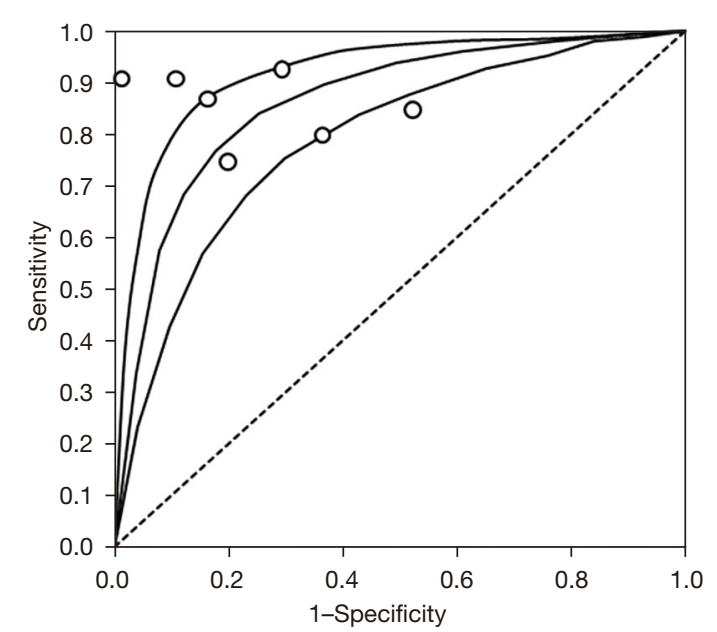

Figure 9 sROC curve of FDG-PET imaging. sROC, summary receiver operating characteristic; FDG-PET, fluorodeoxyglucosepositron-emission computed tomography.

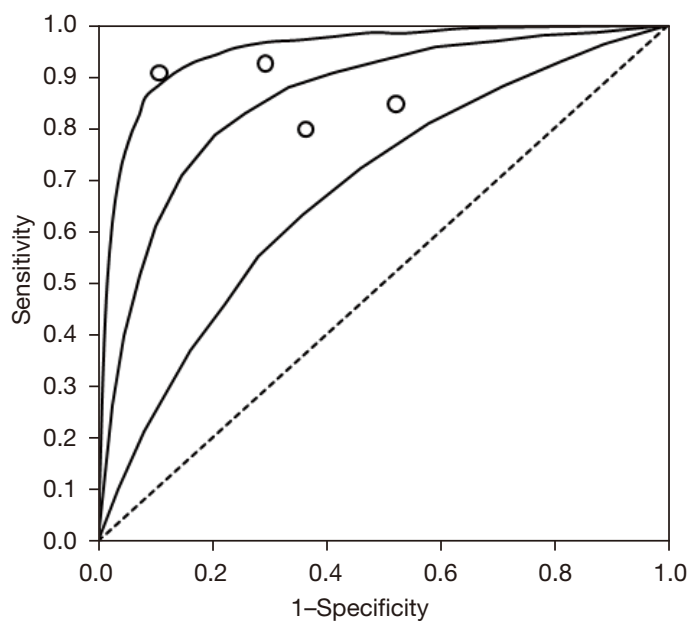

Figure 10 sROC of SPECT imaging. sROC, summary receiver operating characteristic; SPECT, single-photon emission computed tomography. 


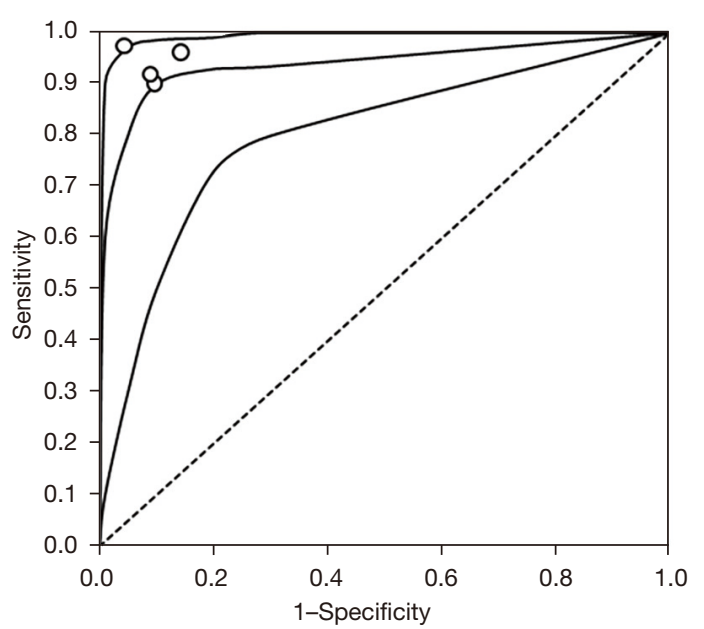

Figure 11 sROC of MRI. sROC, summary receiver operating characteristic; MRI, magnetic resonance imaging.

evaluating cerebral blood perfusion. Rational application of multiple perfusion imaging techniques can provide valuable information for the occurrence mechanism and therapeutic effect evaluation of cognitive dysfunction and other diseases, which is of wide application value.

\section{Conclusions}

This meta-analysis investigated the application value of cerebral perfusion imaging in the prognostic assessment of MCI. The literature was retrieved using the Boolean logic retrieval method, and 16 references concerning FDGPET, SPECT, and MRI imaging were included. The results showed that cerebral perfusion imaging had good prognostic value in patients with MCI, and FDG-PET imaging had superior prognostic ability in patients with MCI.

However, some limitations in this study should be noted. The references included in the study are all case-control studies, and thus, there exists a survival bias. Also, numerous risk factors and indicators were not been included in the study, which greatly reduces the combined effect. In future research, follow-up analysis of patients with MCI should be performed, in order to evaluate the prognostic value of cerebral perfusion imaging for MCI, and to strengthen the findings of this meta-analysis. In conclusion, this study provides a theoretical basis and data support for the clinical diagnosis of MCI and other diseases.v

\section{Acknowledgments}

Funding: None.

\section{Footnote}

Reporting Checklist: The authors have completed the PRISMA-DTA reporting checklist. Available at https://apm. amegroups.com/article/view/10.21037/apm-21-3916/rc

Conflicts of Interest: All authors have completed the ICMJE uniform disclosure form (available at https://apm. amegroups.com/article/view/10.21037/apm-21-3916/coif). The authors have no conflicts of interest to declare.

Ethical Statement: The authors are accountable for all aspects of the work in ensuring that questions related to the accuracy or integrity of any part of the work are appropriately investigated and resolved.

Open Access Statement: This is an Open Access article distributed in accordance with the Creative Commons Attribution-NonCommercial-NoDerivs 4.0 International License (CC BY-NC-ND 4.0), which permits the noncommercial replication and distribution of the article with the strict proviso that no changes or edits are made and the original work is properly cited (including links to both the formal publication through the relevant DOI and the license). See: https://creativecommons.org/licenses/by-nc-nd/4.0/.

\section{References}

1. Faria AL, Andrade A, Soares L, et al. Benefits of virtual reality based cognitive rehabilitation through simulated activities of daily living: a randomized controlled trial with stroke patients. J Neuroeng Rehabil 2016;13:96.

2. Huang C, Mårtensson J, Gögenur I, et al. Exploring Postoperative Cognitive Dysfunction and Delirium in Noncardiac Surgery Using MRI: A Systematic Review. Neural Plast 2018;2018:1281657.

3. Haeger A, Costa AS, Schulz JB, et al. Cerebral changes improved by physical activity during cognitive decline: A systematic review on MRI studies. Neuroimage Clin 2019;23:101933.

4. Basaia S, Agosta F, Wagner L, et al. Automated classification of Alzheimer's disease and mild cognitive 
impairment using a single MRI and deep neural networks. Neuroimage Clin 2019;21:101645.

5. Ottoy J, Niemantsverdriet E, Verhaeghe J, et al. Association of short-term cognitive decline and MCIto-AD dementia conversion with CSF, MRI, amyloidand 18F-FDG-PET imaging. Neuroimage Clin 2019;22:101771.

6. Stephen R, Liu Y, Ngandu T, et al. Brain volumes and cortical thickness on MRI in the Finnish Geriatric Intervention Study to Prevent Cognitive Impairment and Disability (FINGER). Alzheimers Res Ther 2019;11:53.

7. Mosconi L, Perani D, Sorbi S, et al. MCI conversion to dementia and the APOE genotype: a prediction study with FDG-PET. Neurology 2004;63:2332-40.

8. Chételat G, Desgranges B, de la Sayette V, et al. Mild cognitive impairment: Can FDG-PET predict who is to rapidly convert to Alzheimer's disease? Neurology 2003;60:1374-7.

9. Drzezga A, Grimmer T, Riemenschneider M, et al. Prediction of individual clinical outcome in MCI by means of genetic assessment and (18)F-FDG PET. J Nucl Med 2005;46:1625-32.

10. Anchisi D, Borroni B, Franceschi M, et al. Heterogeneity of brain glucose metabolism in mild cognitive impairment and clinical progression to Alzheimer disease. Arch Neurol 2005;62:1728-33.

11. Arnáiz E, Jelic V, Almkvist O, et al. Impaired cerebral glucose metabolism and cognitive functioning predict deterioration in mild cognitive impairment. Neuroreport 2001;12:851-5.

12. Encinas M, De Juan R, Marcos A, et al. Regional cerebral blood flow assessed with $99 \mathrm{~m}$ Tc-ECD SPET as a marker of progression of mild cognitive impairment to Alzheimer's disease. Eur J Nucl Med Mol Imaging 2003;30:1473-80.

13. Borroni B, Anchisi D, Paghera B, et al. Combined 99mTcECD SPECT and neuropsychological studies in MCI for the assessment of conversion to AD. Neurobiol Aging 2006;27:24-31.

14. Hirao K, Ohnishi T, Hirata Y, et al. The prediction of rapid conversion to Alzheimer's disease in mild cognitive impairment using regional cerebral blood flow SPECT. Neuroimage 2005;28:1014-21.

15. Johnson KA, Jones K, Holman BL, et al. Preclinical prediction of Alzheimer's disease using SPECT. Neurology 1998;50:1563-71.

16. Dickerson BC, Goncharova I, Sullivan MP, et al. MRIderived entorhinal and hippocampal atrophy in incipient and very mild Alzheimer's disease. Neurobiol Aging
2001;22:747-54.

17. Duchesne S, Bocti C, De Sousa K, et al. Amnestic MCI future clinical status prediction using baseline MRI features. Neurobiol Aging 2010;31:1606-17.

18. Convit A, de Asis J, de Leon MJ, et al. Atrophy of the medial occipitotemporal, inferior, and middle temporal gyri in non-demented elderly predict decline to Alzheimer's disease. Neurobiol Aging 2000;21:19-26.

19. Devanand DP, Pradhaban G, Liu X, et al. Hippocampal and entorhinal atrophy in mild cognitive impairment: prediction of Alzheimer disease. Neurology 2007;68:828-36.

20. Visser PJ, Verhey FR, Hofman PA, et al. Medial temporal lobe atrophy predicts Alzheimer's disease in patients with minor cognitive impairment. J Neurol Neurosurg Psychiatry 2002;72:491-7.

21. Galton CJ, Erzinçlioglu S, Sahakian BJ, et al. A comparison of the Addenbrooke's Cognitive Examination (ACE), conventional neuropsychological assessment, and simple MRI-based medial temporal lobe evaluation in the early diagnosis of Alzheimer's disease. Cogn Behav Neurol 2005; 18:144-50.

22. Teipel SJ, Born C, Ewers M, et al. Multivariate deformation-based analysis of brain atrophy to predict Alzheimer's disease in mild cognitive impairment. Neuroimage 2007;38:13-24.

23. Train the Brain Consortium. Randomized trial on the effects of a combined physical/cognitive training in aged MCI subjects: the Train the Brain study. Sci Rep 2017;7:39471.

24. Hall JM, Lewis SJG. Neural Correlates of Cognitive Impairment in Parkinson's Disease: A Review of Structural MRI Findings. Int Rev Neurobiol 2019;144:1-28.

25. Meng D, Hosseini AA, Simpson RJ, et al. Large-scale network dysfunction in vascular cognitive disorder supports connectional diaschisis in advanced arteriosclerosis. Eur J Neurol 2020;27:352-9.

26. van Wijnen A, Petrov F, Maiworm M, et al. Cortical quantitative MRI parameters are related to the cognitive status in patients with relapsing-remitting multiple sclerosis. Eur Radiol 2020;30:1045-53.

27. D'Antonio J, Simon-Pearson L, Goldberg T, et al. Cognitive training and neuroplasticity in mild cognitive impairment (COG-IT): protocol for a two-site, blinded, randomised, controlled treatment trial. BMJ Open 2019;9:e028536.

28. Cui Q, Wang D, Zeng M, et al. Association of postoperative covert stroke and cognitive dysfunction among elderly patients undergoing non-cardiac surgery: protocol for a prospective cohort study (PRECISION 
study). BMJ Open 2020;10:e034657.

29. Rane S, Owen J, Hippe DS, et al. White Matter Lesions in Mild Cognitive Impairment and Idiopathic Parkinson's Disease: Multimodal Advanced MRI and Cognitive

Cite this article as: Zhu L, Zhao W, Chen J, Li G, Qu J. Systematic review and meta-analysis of diagnostic test accuracy (DTA) studies: the role of cerebral perfusion imaging in prognosis evaluation of mild cognitive impairment. Ann Palliat Med 2022;11(2):673-683. doi: 10.21037/apm-21-3916
Associations. J Neuroimaging 2020;30:843-50.

(English Language Editor: A. Kassem) 